Pacific Journal of Mathematics

ASYMPTOTIC BEHAVIOR OF MULTIPLICITIES OF 


\title{
ASYMPTOTIC BEHAVIOR OF MULTIPLICITIES OF REPRESENTATIONS OF COMPACT GROUPS
}

\author{
Robert S. CAHN ANd Michael E. TAYlor
}

Let $G$ be a compact Lie group, $K$ a compact subgroup. We denote by $\{\lambda\}$ a complete set of irreducible unitary representations of $G$, which we identify with lattice points within a Weyl chamber $\mathscr{C}$ in $R^{n}$, the cotangent space to a maximal torus in $G$. Similarly we denote by $\{\rho\}$ a complete set of irreducible representations of $K$. The purpose of this paper is to study the asymptotic behavior of the multiplicity $\nu(\rho, \lambda)$ with which $\rho$ is contained in $\lambda$, for fixed $\rho$, as $\lambda \rightarrow \infty$ in $\mathscr{E}$.

We fix some notations and recall a few basic facts. Suppose $G$ is semisimple. If $g_{c}$ is the complexification of the Lie algebra of $G$, then we may write $\mathfrak{g}_{c}=\mathfrak{h}+\sum_{\alpha>0}\left(\mathfrak{g}_{a}+\mathfrak{g}_{-\alpha}\right)$ where $\alpha$ runs through the positive roots of $\mathscr{T}_{c}$ with respect to some fixed order, $\mathfrak{h} \approx C^{k}$ is the Cartan subalgebra of $g_{c}$. We denote $\operatorname{rank} G=\operatorname{dim}_{C} \mathfrak{h}$ by $k$ and the number of positive roots by $b$. The irreducible representations $\{\lambda\}$ of $G$ are naturally identified with certain linear functionals on $\mathfrak{h}_{R}$, called dominant weights, which belong to a lattice $\mathscr{J} \subset \mathfrak{h}_{R}^{*}$, intersected with a Weyl chamber $\mathscr{C}$. If $T^{k}$ is a maximal torus in $G$, then all its representations are one-dimensional, and each one corresponds to some point in $\mathscr{J}$. Further basic results will be recalled in subsequent sections.

Two special cases of our problem are particularly worth mentioning. First, $K$ could be a maximal torus $T^{k}$ in $G$, which we will suppose to be simply connected, semisimple. Then $\{\rho\} \subset \mathcal{F}$. In that case the multiplicity $\nu(\rho, \lambda)$ is given by Kostant's formula

$$
\boldsymbol{\nu}(\rho, \lambda)=\sum_{s \in W}(\operatorname{det} s) P(s(\lambda+\delta)-(\rho+\delta)) .
$$

Here $W$ is the Weyl group, which acts on $\mathscr{J}$, and $\delta=1 / 2 \sum_{\alpha>0} \alpha$ is half the sum of the positive roots, $P(\gamma)$ is a partition function counting the number of distinct ways of writing $\gamma=\sum_{\alpha>0} n_{\alpha} \alpha, n_{\alpha}$ a nonnegative integer. If $\mathscr{R}$ is the lattice generated by the roots, then $\mathscr{R}$ is a sublattice of $\mathscr{F}$. Kostant's formula shows that $\nu(\rho, \lambda)=0$ unless $\lambda-\rho \in \mathscr{R}$ and $-\lambda \leqq \rho \leqq \lambda$. Thus $\nu(\rho, \lambda) \neq 0$ on a set of density at most $\left.|\mathscr{J}| \mathscr{R}\right|^{-1}$. Thus there is wild oscillation in $(0,1)$. It does not appear easy to read off from (0.1) the asymptotic formulas which we will produce. In the second special case of our results, let $H$ be any compact, semisimply simply connected Lie group, with a complete set of irreducible representations $\hat{H}=\{\pi\}$. Let $G=H \times H, K=H^{4}$. 
Clearly the irreducible representation of $G$ are $\left\{\pi_{1} \otimes \pi_{2}: \pi_{j} \in \hat{H}\right\}$, and $\nu\left(\pi, \pi_{1} \otimes \pi_{2}\right)$ are the Clebsch-Gordan numbers for the decomposition of a tensor product of representations

$$
\left.\pi_{1} \otimes \pi_{2}\right|_{H^{\Delta}}=\sum_{\pi \in \hat{H}} \nu\left(\pi, \pi_{1} \otimes \pi_{2}\right) \pi .
$$

In this case there is also a formula for the multiplicities, due to Steinberg, which says

$$
\begin{aligned}
\nu\left(\pi, \pi_{1} \otimes \pi_{2}\right)= & \sum_{S, T \in W_{0}} \operatorname{det}(S T) P\left(S\left(\pi_{1}+\hat{o}_{0}\right)+T\left(\pi_{2}+\delta_{0}\right)\right. \\
& \left.-\left(\pi+2 \delta_{0}\right)\right)
\end{aligned}
$$

where $W_{0}$ is the Weyl group of $H$ and where $\delta_{0}$ is half the sum of the positive roots of $H, P$ is the same partition function as the one in $(0 \cdot 1)$. Again, it is difficult to perceive the asymptotic behavior of the multiplicities from this formula. In this latter case one of our asymptotic formulas has a fairly striking feature. Namely, if $d_{\pi}$ denotes the degree of the representation $\pi$, so the degree of $\lambda=$ $\pi_{1} \otimes \pi_{2}$ is $d_{2}=d_{\pi_{1}} d_{\pi_{2}}$, and if $\hat{G}=\left\{\pi_{1} \otimes \pi_{2}\right\}$ is ordered according to the size of $\left|\pi_{1}\right|^{2}+\left|\pi_{2}\right|^{2}-2\left|\delta_{0}\right|^{2}$, say $\hat{G}=\left\{\lambda_{j}\right\}$, then

$$
\frac{\sum_{j=1}^{N} d_{\lambda_{j}} \nu\left(\pi, \lambda_{j}\right)}{\sum_{j=1}^{N} d_{\lambda_{j}}} \sim C_{H} d_{\pi} N^{-1 / 2} .
$$

Note that the exponent of $N$ in (0.4) is the same for all groups.

Our analysis of the asymptotic behavior of $\nu(\rho, \lambda)$ begins with a result proved in [3]. Namely, if $U$ is an open subset of $\mathscr{C}$ with a decent boundary, $U_{R}=\{R x: x \in U\}$, then

$$
\sum_{\lambda+\hat{o} \in U_{R}} d_{\lambda} \nu(\rho, \lambda) \sim c_{1} \hat{o}_{\rho} R^{n-m}
$$

where $d_{\lambda}$ is the degree of the representation $\lambda, \delta_{\rho}$ is the degree of $\rho, n=\operatorname{dim} G, m=\operatorname{dim} K$, and

$$
c_{1}=c(U, K, G)=(\operatorname{vol} K)^{-1} \mathscr{C}^{n-m}\left(N_{e}^{*}(K) \cap \operatorname{Ad} G(U)\right) .
$$

Here $\mathscr{H}^{\mu}$.denotes $\mu$-dimensional Hausdorff measure, Ad stands for the co-adjoint representation of $G$ on $\boldsymbol{R}^{k}$, and $N_{e}^{*}(K)$ is the conormal space to $K$ at the identity element $e$. We will sketch a proof of (0.5) in $\S 1$, referring to [3] for most of the details. In $\S 2$ we will draw further consequences from (0.5), making use of Weyl's formula for the degree $d_{\lambda}$. It is also desirable to consider the asymptotic behavior of

$$
\sum_{\lambda+\delta \in U_{R}} \nu(\rho, \lambda)
$$


(with the factor $d_{\lambda}$ omitted). We give an example when (0.7) has different asymptotic behavior than one would naively expect from (0.5). In this example, $\nu(\rho, \lambda)$ is supported on the walls of the Weyl chamber $\mathscr{C}$. On the other hand, (0.5) and (0.6) give a geometric interpretation of such a phenomenon. It also gives rise to some questions which we have not answered. The last two sections treat the two special cases we have mentioned, namely the asymptotic behavior of the multiplicities of weights and of the Clebsch-Gordon coefficients.

1. Casimir operators and basic asymptotics. In this section we will discuss (0.5) and some variations, referring to [3] for detailed proofs. The method uses a spectral analysis of the bi-invariant differential operators on $G$, also known as Casimir operators. An example is $\Delta$, the Laplacian on $G$, endowed with a bi-invariant Riemannian metric (which induces a metric on $\boldsymbol{R}^{k}$, the cotangent space to a maximal torus $\boldsymbol{T}^{k}$ ). In general, the entry functions $\pi_{i}^{i j}(x)$ of an irreducible representation $\lambda$ of $G$ belong to an eigenspace of each bi-invariant differential operator $P$, for each fixed $\lambda$. For example, we have

$$
-\Delta \pi_{\lambda}^{i j}=\left(|\lambda+\delta|^{2}-|\delta|^{2}\right) \pi_{i}^{i j} .
$$

The following important theorem describes the action of general Casimir operators.

THEOREM 1.1. Let $q_{m}(\lambda)$ be any homogeneous polynomial on $\boldsymbol{R}^{k}$, of degree $m$, which is invariant under the action of the Weyl group. There exists a bi-invariant differential operator $Q$, of order $m$, such that

$$
Q \pi_{\lambda}^{i j}=q_{m}(\lambda+\delta) \pi_{\lambda}^{i j} .
$$

For a proof, see Zelobenko [4], p. 369. The principal symbol, $q_{m}(e, \xi)$, of $Q$ is described as follows. We think of $\lambda \in T_{e}^{*}\left(\boldsymbol{T}^{k}\right)$ included in $T_{e}^{*}(G)$, and then $q_{m}(\lambda)=q_{m}(e, \lambda)$. Since $q_{m}(e, \xi)$ is invariant under the adjoint action of $G$ on $T_{e}^{*}(G)$, this uniquely specifies $q_{m}(e, \xi)$.

We remark that, given any Casimir operator $P, P \pi_{\lambda}^{i j}=p(\lambda) \pi_{i}^{i j}$ where $p(\lambda)$ is a polynomial whose leading term is $p_{m}(e, \lambda)$, where $p_{m}(x, \xi)$ is the principal symbol of $P$. It seems to be a major outstanding problem in the theory of compact Lie groups to completely specify $p(\lambda)$. For the same reason, one does not know the complete symbol of the operator $Q$ satisfying (1.2).

As a consequence of Theorem 1.1 and a functional calculus, the following result is proved in [3]. 
THeOREM 1.2. Let $p(\lambda) \in S_{1,0}^{m}\left(\boldsymbol{R}^{k}\right)$ be invariant under the Weyl group. Let $P: \mathscr{D}^{\prime}(G) \rightarrow \mathscr{D}^{\prime}(G)$ be defined by

$$
P \pi_{\lambda}^{i j}=p(\lambda+\delta) \pi_{\lambda}^{i j} .
$$

Then $P \in O P S_{1,0}^{m}$ on $G$.

Here $S_{1.0}^{m}$ denotes the symbol class of Hörmander [1]. Generally, $p(x, \xi) \in S_{1,0}^{m}$ provided

$$
\left|D_{x}^{\beta} D_{\xi}^{\alpha} p(x, \xi)\right| \leqq C_{\alpha \beta}(1+|\xi|)^{-|\alpha|+m} .
$$

The conclusion is that $P=p(x, D)$ is a pseudo differential operator on $G$. The principal symbol of $P$ is specified by the relation $p_{m}(e, \lambda)=$ $p(\lambda) \bmod S_{1,0}^{m-1}$. This result will be applied to a one parameter family of functions $p_{t}(\lambda)=p(t \lambda)$, given $p(\lambda) \in C_{0}^{\infty}\left(R^{k}\right)$, which we think of as a bounded subset of $S_{1,0}^{0}\left(R^{k}\right)$.

The way Theorem 1.2 enters into the study of the multiplicities $\nu(\rho, \lambda)$ is the following. By the Weyl orthogonality relations, one sees that

$$
\delta_{\rho} \int_{K} \lambda(x) \operatorname{tr} \rho(x) d x=P_{\lambda, \rho}
$$

is the projection onto the subspace of $V_{\lambda}$, the representation space of $\lambda$, on which $K$ acts like copies of $\rho$. Since $\delta_{\rho} \nu(\rho, \lambda)$ is easily seen to be the square of the Hilbert-Schmidt norm of $P_{\lambda, \rho}$, we have

$$
\begin{aligned}
\nu(\rho, \lambda) & =\delta_{\rho} \sum_{i, j}\left|\int_{K} \operatorname{tr} \rho(x) \pi_{\lambda}^{i,}(x) d x\right|^{2} \\
& =\frac{\delta_{\rho}}{d_{\lambda}} \sum_{i, j}\left|\left((\operatorname{tr} \rho) \mu_{K}, \sqrt{\bar{d}_{\lambda}} \pi_{i}^{i j}\right)\right|^{2}
\end{aligned}
$$

where $\mu_{K}$ is Haar measure on $K$, considered as a measure on $G$. Consequently, if $p_{1}$ is a sufficiently rapidly decreasing function on $\boldsymbol{R}^{k}$ we have

$$
\begin{aligned}
\sum_{i \in \mathcal{Y} \cap \mathscr{E}} d_{\lambda} \nu(\rho, \lambda) p_{1}(\lambda) & =\delta_{\rho} \sum_{\lambda} \sum_{i, j} p_{1}(\lambda)\left|\left((\operatorname{tr} \rho) \mu_{K}, \sqrt{d_{\lambda}} \pi_{\lambda}^{i j}\right)\right|^{2} \\
& =\delta_{\rho}\left(P_{1} \mu, \mu\right)
\end{aligned}
$$

where $\mu=(\operatorname{tr} \rho) \mu_{K}$ and the operator $P_{1}$ is defined by

$$
P_{1} \pi_{i}^{i j}=p_{1}(\lambda) \pi_{i}^{i j} .
$$

We suppose $p_{1}(\lambda)=p(\lambda+\delta)$ with $p(\lambda) \in S_{1,0}^{-s}\left(R^{k}\right)$ invariant under the Weyl group, $s>n-m$ (recall that $n=\operatorname{dim} G, m=\operatorname{dim} K$ ). We can replace $p(\lambda)$ by $p(t \lambda)$. Analyzing the operator $P_{t}$ defined by $P_{t} \pi_{\lambda}^{i j}=$ $p(t(\lambda+\delta)) \pi_{i}^{i j}$ as a pseudo differential operator, we can describe the 
behavior of $\left(P_{t} \mu, \mu\right)$ as $t \rightarrow 0$. The idea behind this, briefly sketched, is the following. The leading term in $P_{t}$ is $\widetilde{p}_{m}\left(x, t D_{x}\right)$, which is an operator defined by a Poisson like kernel,

$$
p_{m}\left(x, t D_{x}\right) u=\int K_{t}(x, x-y) u(y) d y
$$

where

$$
K_{t}(x, x-y)=\int \widetilde{p}_{m}(x, t \xi) e^{i(x-y) \cdot \xi} d \xi
$$

From this follows that

$$
\begin{aligned}
\left(P_{t} \mu, \mu\right) & =\int K_{t}(x, x-y) d \mu(y) \overline{d \mu(x)} \\
& \sim t^{-(n-k)}(\operatorname{vol} K)^{-2} \int_{N^{*}(K)}|\operatorname{tr} \rho(x)|{ }^{2} \widetilde{p}_{m}\left((d x, \xi) d \operatorname{vol}_{N^{*}(K)}(x, \xi)\right. \\
& =t^{-(n-k)}(\operatorname{vol} K)^{-1} \int_{N_{e}^{*}(K)} \widetilde{p}_{m}(e, \xi) d \operatorname{vol}_{N_{e}^{*}(K)}(\xi),
\end{aligned}
$$

since the bi-invariant operator $P$ has a bi-invariant symbol $\widetilde{p}_{m}$, and $\int_{K}|\operatorname{tr} \rho(x)|^{2} d x=1$ by the irreducibility of $\rho$. The symbol $\widetilde{p}_{m}(e, \xi)$ is described in analogy to the symbols of the Casimir operators. Namely $\widetilde{p}_{m}(e, \lambda)=p(\lambda)$ and $\widetilde{p}_{m}(e, \xi)$ is invariant under the adjoint action of $G$. For further details, see [3].

Combining this analysis of $\left(P_{t} \mu, \mu\right)$ with (1.4) yields the following (taking $R=t^{-1}$ ).

THEOREM 1.3. $\sum_{\lambda} d_{\lambda} \nu(\rho, \lambda) p\left(R^{-1}(\lambda+\delta)\right) \sim c_{0} \delta_{\rho} R^{n-m}, R \rightarrow \infty$, where

$$
c_{0}=(\operatorname{vol} K)^{-1} \int_{N_{e}^{*}(K)} p(F(\xi)) d \operatorname{vol}_{N_{e}^{*}(K)}(\xi) \text {. }
$$

Here we define $F: R^{n} \rightarrow R^{n} / W$ by $F(\xi)=$ any $\lambda \in R^{k}$ such that $\xi=\operatorname{Ad}(x) \lambda$ for some $x \in G$. In fact, one can obtain a complete asymptotic expansion in decreasing powers of $R$ (and $\log R$ terms). Lower order coefficients may also contain interesting geometric quantities. However, due to the unresolved nature of the precise eigenvalues of the Casimir operators in general, we cannot analyze these coefficients presently. In the special case when $p(\lambda)$ is radial, so $P_{t}$ is a function of the Laplace operator, one could evaluate the next couple of coefficients, but we shall not do so. Note that we must distinguish between $d \operatorname{vol}_{K}$, induced by the Riemannian metric on $G$ (which makes vol $G=1$ ) and Haar measure on $K$, which assigns to $K$ a total mass of $1 ; d \operatorname{vol}_{K}=(\operatorname{vol} K) \mu_{K}$. If $K$ is discrete, for example, vol $K=0(K)$. 
Let $V$ be a Weyl group invariant bounded open subset of $\boldsymbol{R}^{k}$, whose boundary has $\omega$ measure zero, where the measure $\omega$ is defined by

$$
\int_{R^{\prime t}} p(\lambda) d \omega(\lambda)=\int_{N_{e}^{*}(K)} p(F(\xi)) d \operatorname{vol}_{N_{e}^{*}(K)}(\xi) .
$$

Then we can approximate the characteristic function of $V$ nicely by smooth functions with compact support and pass to the limit. Since if $p(\lambda)$ is the characteristic function of $V$, then $p(F(\xi))$ is the characteristic function of the orbit $\operatorname{Ad} G(V)$ of $V$ under $\operatorname{Ad} G$, in $\boldsymbol{R}^{n}=T_{e}^{*}(G)$, Theorem 1.3 leads upon passing to the limit to the following. We set $U=V \cap \mathscr{C}$.

THEOREM 1.4. With notations as above, and $U_{R}=\{R \lambda: \lambda \in U\}$, we have

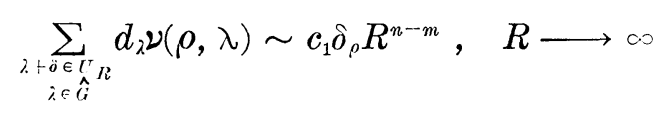

where

$$
c_{1}=(\operatorname{vol} K)^{-1} \operatorname{vol}\left(N_{e}^{*}(K) \cap \operatorname{Ad} G(U)\right) .
$$

This is the result (0.5) mentioned in the introduction.

2. Mean behavior of multiplicities. In this section we examine some consequences of Theorem 1.3, relating in particular to the mean frequency with which a given representation $\rho$ of $K$ is contained in the various representations of $G$. Thus we are interested in the asymptotic behavior of

$$
\frac{\sum_{\lambda+\dot{j} \in U_{R}} d_{\lambda} \nu(\rho, \lambda)}{\sum_{\lambda+\dot{o} \in U_{R}} d_{\lambda}}=F_{U}(R) .
$$

In this case, we are weighting the $\nu(\rho, \mu)$ in terms of the dimension $d_{\lambda}$ of the representation space on which $\lambda$ acts. This biasing is natural if we interpret $\delta_{\rho} d_{\lambda} \nu(\rho, \lambda)$ as the dimension of the subspace of $L^{2}(G)$ where $K$ acts like copies of $\rho$, and regard it as the natural object of study, and of course it was this perspective which produced Theorem 1.3. Nevertheless, it is also interesting to consider the asymptotic behavior of

$$
\frac{\sum_{\lambda+\hat{o} \in U_{R}} \nu(\rho, \lambda)}{\sum_{\lambda+\hat{o} \in U_{J_{l}}} 1}=F_{U}^{\prime}(R) .
$$


Our analysis of (2.2) will be less complete than the analysis of (2.1). In order to analyze $F_{L}(R)$, in terms of $(0.5)$, we use Weyl's formula

$$
d_{\lambda}=\phi(\lambda+\delta)=\frac{\prod_{\alpha>0}\langle\lambda+\delta, \alpha\rangle}{\prod_{\alpha>0}\langle\delta, \alpha\rangle} .
$$

Thus $\phi(\lambda)$ is a homogeneous polynomial of degree $b$, where $b$ is the number of positive roots of $G$. We remark that, if $S$ is an element of the Weyl group, then $\phi(S \lambda)=(-1)^{l(S)} \phi(\lambda)$, where $l(S)=$ the number of reflections into which $S$ factors $(\bmod 2)$. We see that

$$
\sum_{\lambda \rightarrow+j \in U^{*} R} d_{\lambda} \sim \int_{V^{*} R} \phi(\lambda) d \lambda=c_{2} R^{k+b},
$$

while

$$
\sum_{\lambda+\delta \in U_{R}} 1 \sim c_{3} R^{k}
$$

From (2.4) and Theorem 1.4 we get the following result, using the identity

$$
n=k+2 b
$$

THEOREM 2.1. $F_{V}(R) \sim c_{1} c_{2}^{-1} \delta_{\rho} R^{b-m}$.

We can analyze (2.2) in the case where $U$ is bounded away from the walls of the Weyl chamber $\mathscr{C}$. In fact, with $\mathrm{X}(\lambda) \in C_{0}^{\infty}(C)$ approximately equal to the characteristic function $\mathrm{X}_{U}$ of $U$, apply Theorem 1.3 with $p(\lambda)=\phi(\lambda)-\mathrm{X}(\lambda)$. Since $\phi(\lambda)$ is homogeneous of degree $b$ we get

$$
p\left(R^{-1}(\lambda+\delta)\right)=R^{b} \phi(\lambda+\delta)^{-1} \mathrm{X}\left(R^{-1}(\lambda+\delta)\right)=R^{b} d_{\lambda}^{-1} \mathrm{X}\left(R^{-1}(\lambda+\delta)\right) .
$$

Passing to the limit yields the following.

THEOREM 2.2. Provided $U$ is bounded away from the walls of $\mathscr{C}$, we have

$$
F_{U}^{\prime}(R) \sim c_{4} c_{3}^{-1} \delta_{\rho} R^{b-m}
$$

where

$$
c_{4}=(\operatorname{vol} K)^{-1} \int_{N_{e}^{*}(K)} \phi(F(\xi))^{-1} \mathrm{X}_{L}(F(\xi)) d \operatorname{vol}_{N_{e}^{*}(K)}(\xi)
$$

The hypothesis that $U$ be bounded away from the walls of $\mathscr{C}$ is necessary for the above analysis because $\phi(\lambda)^{-1}$ blows up at the 
walls. Note that the exponents of $R$ in Theorems 2.1 and 2.2 are the same in this case. For sets $U$ which intersect the walls $\mathscr{C}$, $F_{U}(R)$ and $F_{U}^{\prime}(R)$ may under some circumstances have different asymptotic behavior.

Indeed, consider ther following examples. Suppose $(G, K)$ is a Riemannian symmetric pair, so $G / K$ is a symmetric space. We have a splitting of the Lie algebra of $G, \mathfrak{g}=\mathfrak{f}+\mathfrak{p}$. Let $\mathfrak{a}_{\mathfrak{p}}$ be a maximal abelian subspace of $\mathfrak{p}$. By definition, $\operatorname{dim} \mathfrak{a}_{\mathfrak{p}}$ is the $\operatorname{rank}$ of $G / K$; denote this integer by $\kappa$. Note that if $a$ is a maximal abelian subspace of $\mathfrak{g}$ containing $\mathfrak{a}_{\mathfrak{p}}$ then $\mathfrak{a}$ is the Lie algebra of a maximal torus $\boldsymbol{T}^{k}$, so $k \geqq \kappa$. Write $\mathfrak{a}=\mathfrak{a}_{k}+\mathfrak{a}_{\mathfrak{p}}$. The following important result is due to E. Cartan.

THEOREM 2.3. $L^{2}(G / K) \approx \sum_{\pi \in \hat{G}_{K}} V_{\pi}$ where $V_{\pi}$ is the representation space of $\pi$, and

$$
\widehat{G}_{K}=\{\pi \in \hat{G}: \exists v \quad \text { with } \quad \pi(K) v=v\} .
$$

Furthermore, $\lambda \in \hat{G}$ belongs to $\hat{G}_{K}$ if and only if $\lambda\left(a_{\mathfrak{p}}\right)=0$.

In particular, $\lambda \in \hat{G}$ belongs to $\hat{G}_{K}$ if and only if $\nu\left(1_{K}, \lambda\right) \neq 0$, and in that case, we have $\nu\left(1_{K}, \lambda\right)=1$. $\widehat{G}_{K}$ belongs to a sublattice $\mathscr{J}_{1}$ of $\mathscr{J}$, satisfying the relation $\lambda\left(\mathfrak{a}_{\mathfrak{p}}\right)=0$, which is a nontrivial relation provided $\operatorname{rank} G>\operatorname{rank} G / K$. If $U$ is the unit ball, intersected with $\ddot{\mathscr{C}}$, we see that, $\rho=1_{K}$,

$$
F_{U}(R) \sim c_{1} c_{2}^{-1} R^{b-m}
$$

while $\sum_{\lambda+\grave{o} \in U_{R}} \nu\left(1_{K}, \lambda\right)=\sum_{\lambda+\grave{o} \in U_{R}, \lambda \in \mathcal{F}_{1}} 1 \sim c_{5} R^{\kappa}$ yields

$$
F_{U}^{\prime}(R) \sim c_{5} c_{3}^{-1} R^{\kappa-k} .
$$

In the case where $\operatorname{rank} G=\operatorname{rank} G / K$, the two exponents in (2.7) and (2.8) agree; $b-m=\kappa-k=0$. However, if $k>\kappa$, these exponents may differ. For example, in the case $G=S O(2 \nu), K=$ $S O(2 \nu-1)$, where $G / K=S^{2 \nu-1}$, we have $k=\nu, \kappa=1$ while $b=$ $\nu(\nu-1), m=(\nu-1)(2 \nu-1)$, so

$$
F_{U}(R) \sim c_{1} c_{2}^{-1} R^{-(\nu-1)(\nu-1)}, \quad F_{U}^{\prime}(R) \sim c_{5} c_{3}^{-1} R^{-(\nu-1)} .
$$

Clearly the exponents differ, for $\nu \geqq 3$.

The reason for the different decay rates in (2.9) is explained simply. The condition $\lambda\left(\mathfrak{a}_{p}\right)=0$ implies that the element of $\hat{G}_{K}$ are contained in a wall of the Weyl chamber $\mathscr{C}$, indeed, perhaps even in a corner. As the Weyl formula shows, $d_{\lambda}$ is relatively small near the walls. If it is known that $\nu(\rho, \lambda)$ is nonzero only on a corner of the Weyl chamber of codimension $j$, but $\nu(\rho, \lambda)$ is supported away 
from corners of codimension $j+1$, one can work out an asymptotic relation analogous to Theorem 2.2, with a different exponent.

Obviously, if $\nu(\rho, \lambda)$ is supported on the walls of $\mathscr{C}$, and if $U$ is chosen disjoint from $\partial \mathscr{C}, F_{U}(R) \equiv 0$, so the constant $c_{1}$ of Theorem 1.4 vanishes. Consequently, if we write $\overline{\mathscr{C}}_{1}=\overline{\mathscr{C}} \cap\{$ open unit ball as $\overline{\mathscr{C}}_{1}=\dot{\mathscr{C}}_{1} \cup(\partial \mathscr{C} \cap\{$ unit ball $\})$, formula (1.8) implies the following.

Proposition 2.4. If $\nu(\rho, \lambda)$ is supported on the walls of $\mathscr{C}$, then

$$
\operatorname{vol}\left(N_{e}^{*}(K) \cap \operatorname{Ad} G\left(\dot{\mathscr{C}}_{1}\right)\right)=0 .
$$

More generally, if $\nu(\rho, \lambda)$ is supported in a corner $\mathscr{K}$, and you decompose $\overline{\mathscr{C}}_{1}$, as $\overline{\mathscr{C}}_{1}=\left(\overline{\mathscr{C}}_{1}-\mathscr{\mathscr { C }}\right) \cup\left(\overline{\mathscr{C}}_{1} \cap \mathscr{K}\right)$, then

$$
\operatorname{vol}\left(N_{e}^{*}(K) \cap \operatorname{Ad} G\left(\overline{\mathscr{C}}_{1}-\mathscr{\mathscr { C }}\right)\right)=0 .
$$

We would conjecture a converse to this result, namely that if (2.11) holds, then $\nu(\rho, \lambda)$ is supported in a lattice parallel to the corner $\mathscr{K}$. Note that (2.11) implies that, apart from a set of measure zero in $N_{e}^{*}(K)$, the entire intersection $N_{e}^{*}(K) \cap \operatorname{Ad} G\left(\overline{\mathscr{C}}_{1}\right)$ is equal to $N_{e}^{*}(K) \cap \operatorname{Ad} G\left(\overline{\mathscr{C}}_{1} \cap \mathscr{K}\right)$. This seems to be an interesting geometric phenomenon related to the representation theory of $G$ and $K$.

Finally, we mention one other measure of the mean behavior of $\nu(\rho, \lambda)$. Namely, let $\Phi(N)=F_{U}(R)$ where $U$ is the unit ball $(\cap \overline{\mathscr{C}})$ and $R$ is chosen so there are $N$ elements of $\mathscr{J}$ in $U_{R}$. Thus we are simply using the total number of representations, ordered by highest weight, as a measure of size, rather than $R$. Theorem 2.1 yields

$$
\Phi(N) \sim c_{6} \delta_{\rho} N^{(b-m) / t}
$$

3. Multiplicities of weights. As was remarked in the introduction one of most interesting cases to consider is when $\mathrm{K}=T^{k}$, a maximal torus. Since $\mathrm{K}$ is abelian each irreducible representation is one-dimensional and each irreducible represention of $G$ splits, when restricted to $\mathrm{K}$, into a direct sum of one-dimensional subrepresentations. The representations of $\mathrm{K}, \rho$, will be identified via the exponential map with linear functionals $\rho$ belonging to $\mathscr{f}$, the lattice of dominant weights. $\mathscr{Y}$ will contain as a sub-lattice of equal rank the root lattice $\mathscr{R}$. For a fixed weight $\rho$, the structure theory of semi-simple Lie algebras gives $\nu(\rho, \lambda)=0$ a prior $i$ unless $-\lambda \leqq \rho \leqq \lambda$ and $\lambda-\rho \in \mathscr{R}$. If both of these conditions hold then

$$
\nu(\rho, \lambda)=\sum_{\mathrm{s} \in W}(\operatorname{det} s) P(s(\lambda+\delta)-(\rho+\delta))
$$

with the notation as in the introduction. 
Formula (3.1) is difficult to work with for two reasons. The partition function is rather unwieldy in even small rank for $\lambda$ of any size and the situation is further obscured by the cancellation produced by the sign of det $s$. In particular the asymptotic behavior of (3.1) is unclear even if we restrict ourselves to $\lambda$ going to infinity along a ray passing through the origin. However, we are able to state the following results about the average size of $\nu(\rho, \lambda)$.

Theorem 3.2. Let $\left\{\lambda_{j}\right\}=\hat{G}$ ordered by the eigenvalue of the Laplace operator; then

$$
\frac{\sum_{i=1}^{N} d_{\lambda_{i}} \nu(\rho, \lambda)}{\sum_{i=1}^{N} d_{\lambda_{i}}} \sim C_{G} N^{(b-k) / k}
$$

Proof. First we remark that since all Cartan subgroups are conjugate that the constant $C_{G}$ is independent of the maximal torus chosen. Secondly, we note that $d_{\rho}=1$ for all $\rho$. Lastly, we note that (2.12) finishes the proof.

It is desirable to also have an average multiplicity without the factors $d_{\lambda}$. We will be able to state a theorem for the average size of $\nu(\rho, \lambda)$ but we will have to restrict ourselves to representations whose dominant weights are sufficiently nonsingular.

THEOREM 3.3. Let $U$ be a bounded open domain in $\mathscr{C}$ such that the $\omega$ measure of $\partial U=0$ and $\partial U \cap \partial \mathscr{C}=\varnothing$ and let $U_{R}$ be $U$ dilated by a factor $R$. Then

$$
F_{l}^{\prime}(R)=\frac{\sum_{i+\dot{\delta} \in U I R} \nu(\rho, \lambda)}{\sum_{\lambda+\dot{\delta} \in U_{R}} 1} \sim c_{4} c_{3}^{-1} R^{b-k} .
$$

We note this is just Theorem 2.2 restated in our setting.

4. Clebsch-Gordon coefficients and induced representations.

If $H$ is a compact Lie group we may take a tensor product of two or more representations of $H$ to create a new, generally reducible, representation. Since $H$ is compact complete reducibility allows us to write such a tensor product as a direct sum of irreducible representations. We will be interested in the multiplicity of a fixed representation $\pi$ in $\pi_{1} \otimes \pi_{2}$.

Let us start by examining the simplest case. If $H=\boldsymbol{R} / 2 \pi \boldsymbol{Z}$ then $\hat{H} \cong \boldsymbol{Z}$. A tensor product is irreducible, i.e., $e^{i n_{1} x} \otimes e^{i n_{2} x}=$ $e^{i\left(n_{1}+n_{2}\right) x}$. If $\pi$ is the trivial representation then $e^{i n_{1} x} \otimes e^{i n_{2} x}=\pi$ when $n_{1}=-n_{2}$. Thus if $\lambda=\left(n_{1}, n_{2}\right) \nu(\pi, \lambda)=1$ on the line through the 
origin of slope -1 and is 0 in all other cases. The eigenvalue of the Casimir operator on the tensor product is $n_{1}^{2}+n_{2}^{2}$ so if we order the tensor products by the eigenvalue we see $\sum_{i=1}^{N} \nu\left(\pi ; \pi_{1} \otimes \pi_{2}\right) \sim 2 / \sqrt{\pi} N^{1 / 2}$. The average value of $\nu\left(\pi, \pi_{1} \otimes \pi_{2}\right)$ in the first $N$ representations is $2 / \sqrt{\pi} N^{-1 / 2}$. The original Clebsch-Gordon coefficients were determined for the case of $S U(2)$. Here the rank is 1 so for each half integer $j$ there exists a unique irreducible representation of dimension $2 j+$ $1, \pi_{j}$, corresponding to the weight $j \lambda$. The tensor product is decomposable by the Clebsch-Gordon series as

$$
\pi_{j} \otimes \pi_{k} \cong \pi_{j+k} \oplus \pi_{j+k-1} \oplus \cdots \oplus \pi_{i j-k \mid}
$$

so one sees $\nu\left(\pi_{i}, \pi_{j} \otimes \pi_{k}\right)=1$ if $j+k-i \in \boldsymbol{Z}|j-k| \leqq i \leqq j+k$ and zero otherwise. One may directly compute the average behavior of $\nu\left(\pi_{i}, *\right)$ but we will instead make a computation which holds for all the semi-simple groups.

As in the case of the multiplicity of a weight there is an exact formula for the Clebsch-Gordon coefficient $\nu\left(\pi, \pi_{1} \otimes \pi_{2}\right)$, for semi-simple groups, due to Steinberg. Keeping the notation as before,

$$
\nu\left(\pi, \pi_{1} \otimes \pi_{2}\right)=\sum_{S, T \in W_{0}} \operatorname{det}(S T) P\left(S\left(\pi_{1}+\hat{\delta}_{0}\right)+T\left(\pi_{2}+\delta_{0}\right)-\left(\pi+2 \delta_{0}\right)\right) .
$$

The difficulties in asymptotically evaluating this formula are the same as in Kostant's formula.

We will now use the machinery of $\S 2$ to analyze the average behavior of the Clebsch-Gordon coefficients. Let $G=H \times H$ with $H$ compact, and simply connected. Let $H^{4}$ be the diagonal subgroup. Then

$$
\pi_{1} \otimes \pi_{2} \mid H^{\lrcorner}=\sum_{\pi \in \hat{I}} \nu\left(\pi, \pi_{1} \otimes \pi_{2}\right) \pi
$$

The set $\left\{\pi_{1} \otimes \pi_{2} \mid \pi_{i} \in \hat{H}\right\}=\hat{G}$. The two copies of $H$ are orthogonal so the eigenvalue of the Laplace operator is $\left|\pi_{1}\right|^{2}+\left|\pi_{2}\right|^{2}-2\left|\delta_{0}\right|^{2}$ and $d_{\pi_{1} \otimes \pi_{2}}=d_{\pi_{1}} d_{\pi_{2}}$. We will order $\hat{G}$ by the eigenvalue and call the $j$ th product $\lambda_{j}$. Since the rank of $G$ is $2 k$ we can apply (2.12) to find

$$
\Phi(N)=\frac{\sum_{j=1}^{N} d_{\lambda_{j} \nu}\left(\pi, \lambda_{j}\right)}{\sum_{j=1}^{N} d_{\lambda_{j}}} \sim C_{l l} d_{\pi} N^{(2 b-n) / 2 k}
$$

but since $n=2 b+k$ we have

THEOREM 4.1. If $H$ any simply connected semi-simple compact Lie group 


$$
\frac{\sum_{i=1}^{N} d_{\lambda_{i}} \nu\left(\pi, \lambda_{i}\right)}{\sum_{i=1}^{N} d_{\lambda_{i}}} \sim C_{H} d_{\pi} N^{-1 / 2}
$$

We conclude with a word about $b$, the critical exponent. As we remarked in $\S 2$ the asymptotic behavior of $\sum_{\lambda+\hat{o} \in U_{R}} \delta_{\rho} \nu(\rho, \lambda) d_{\lambda}$ measures the part of $L^{2}(G)$ corresponding to $U_{R}$ which when we restrict to the action of the subgroup $K$ transforms by $\rho$. The function $F_{U}(R)$ can be thought of as the weighted mean of the multiplicities $\nu(\rho, \lambda)$. Let us look at the case when $\rho=\mathrm{id}$. Then Frobenius reciprocity for compact groups states that the multiplicity of $\lambda$ in $L^{2}(G / \mathrm{K})$ equals $\nu\left(\mathrm{id}_{K}, \lambda\right)$. Thus we have

THEOREM 4.2. If $\operatorname{dim} \mathrm{K}>b$ then most representations of $G$ do not occur in $L^{2}(G / K)$ mean sense.

For an arbitrary $\rho: K \rightarrow \operatorname{Aut}\left(V_{\rho}\right)$ we may form the associated homogeneous vector bundle $E_{\rho}: G \times_{K} V_{\rho} \rightarrow G / K . \quad L^{2}\left(E_{\rho}\right)$, the space of $L^{2}$-sections, is naturally a $G$-module and again reciprocity gives

THEOREM 4.3. If $\operatorname{dim} \mathrm{K}>b$ then most representation of $G$ do not occur in $L^{2}\left(E_{\rho}\right)$ mean sense.

\section{REFERENCES}

1. L. Hörmander, Pseudo differential operators and hypoelliptic equations, Proc. Symp. Pure Math., Vol. X, 138-183.

2. Samelson, Notes on Lie Algebras, Van Nostrand Reinhold, 1969.

3. M. Taylor, Pseudo differential operators, Vol. 2, to appear.

4. D. Zelobenko, Compact Lie groups and their representations, Transl. of Math. Monographs, vol. 40, Amer. Math. Soc., 1973.

Received June 1, 1978, Research by the first author was partially supported by NSF grant MCS 76-03939. Research by the second author was partially supported by Alfred P. Sloan fellowship and by NSF grant MCS 77-03634.

UNIVERSITY OF CALIFORNIA

BERKELEY, CA 94720

UNIVERSITY OF MIANI

Coral Gables, FL 33124

AND

RICE UNIVERSITY

Houston, TX 77001 


\section{PACIFIC JOURNAL OF MATHEMATICS}

\section{EDITORS}

DONALD BABBITT (Managing Editor)

University of California

Los Angeles, California 90024

Hugo RossI

University of Utah

Salt Lake City, UT 84112

C. C. MOORE and ANDrew OGG

University of California

Berkeley, CA 94720
J. DUgundjI

Department of Mathematics University of Southern California Los Angeles, California 90007

R. FinN aNd J. Milgram Stanford University Stanford, California 94305

\section{ASSOCIATE EDITORS}

E. F. BECKENBACH

B. H. NeUMANN

F. WOLF

K. YosHIDA

\section{SUPPORTING INSTITUTIONS}

UNIVERSITY OF BRITISH COLUMBIA CALIFORNIA INSTITUTE OF TECHNOLOGY UNIVERSITY OF CALIFORNIA MONTANA STATE UNIVERSITY UNIVERSITY OF NEVADA, RENO NEW MEXICO STATE UNIVERSITY OREGON STATE UNIVERSITY UNIVERSITY OF OREGON
UNIVERSITY OF SOUTHERN CALIFORNIA STANFORD UNIVERSITY UNIVERSITY OF HAWAII UNIVERSITY OF TOKYO UNIVERSITY OF UTAH WASHINGTON STATE UNIVERSITY UNIVERSITY OF WASHINGTON 


\section{Pacific Journal of Mathematics}

\section{Vol. 84 , No. 1 \\ May, 1979}

Michael James Beeson, Goodman's theorem and beyond ...............

Robert S. Cahn and Michael E. Taylor, Asymptotic behavior of multiplicities

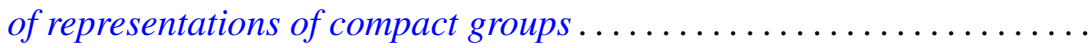

Douglas Michael Campbell and Vikramaditya Singh, Valence properties of

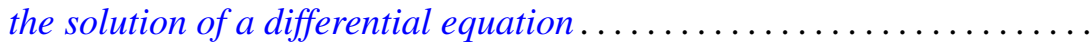

J.-F. Colombeau, Reinhold Meise and Bernard Perrot, A density result in spaces of Silva holomorphic mappings .....................

Marcel Erné, On the relativization of chain topologies .................

Le Baron O. Ferguson, Uniform and $L_{p}$ approximation for generalized

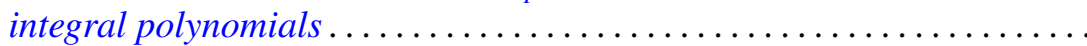

Kenneth R. Goodearl and David E. Handelman, Homogenization of regular rings of bounded index..................................

Friedrich Haslinger, A dual relationship between generalized

Abel-Gončarov bases and certain Pincherle bases .................

Miriam Hausman, Generalization of a theorem of Landau. .

Makoto Hayashi, 2-factorization in finite groups

Robert Marcus, Stochastic diffusion on an unbounded domain ........... 143

Isabel Dotti de Miatello, Extension of actions on Stiefel manifolds....

C. David (Carl) Minda, The hyperbolic metric and coverings of Riemann surfaces...

Somashekhar Amrith Naimpally and Mohan Lal Tikoo, On $T_{1}$-compactifications.

Chia-Ven Pao, Asymptotic stability and nonexistence of global solution for a semilinear parabolic equation ....

Shigeo Segawa, Harmonic majoration of quasibounded type ...

Sze-Kai Tsui and Steve Wright, The splitting of operator algebras ...

Bruce Williams, Hopf invariants, localization and embeddings of Poincaré complexes....

Leslie Wilson, Nonopenness of the set of Thom-Boardman maps ...

Alicia B. Winslow, There are $2^{\mathrm{c}}$ nonhomeomorphic continua in $\beta R^{n}-R^{n}$ 